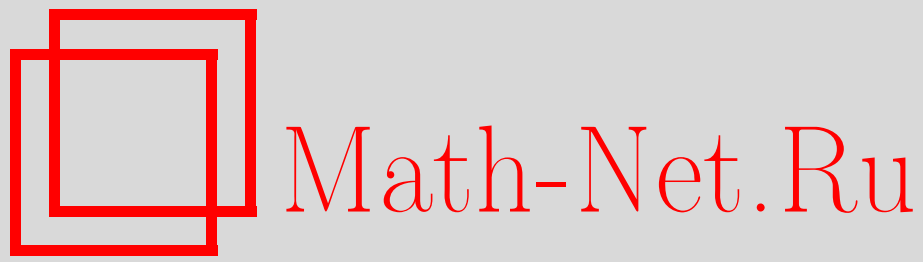

А. А. Шкаликов, Диссипативные операторы в пространстве Крейна. Инвариантные подпространства и свойства сужений, Функи. анализ и его прил., 2007, том 41, выпуск 2, 93-110

DOI: https://doi.org/10.4213/faa2863

Использование Общероссийского математического портала MathNet.Ru подразумевает, что вы прочитали и согласны с пользовательским соглашением

http://www . mathnet.ru/rus/agreement

Параметры загрузки:

IP : 54.224 .135 .184

26 апреля 2023 г., 18:08:11

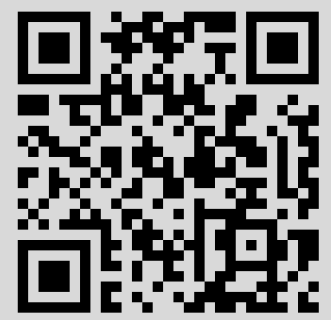




\title{
Диссипативные операторы в пространстве Крейна. Инвариантные подпространства и свойства сужений*
}

\author{
(c) 2007. А. А. ШкАликов
}

К столетию Марка Григоръевича Крейна

\section{$\S 1$. Введение}

Напомним основные определения теории пространств с индефинитной метрикой. Пусть $\mathscr{H}$ - гильбертово пространство, в котором наряду с обычным скалярным произведением $(x, y)$ задано индефинитное скалярное произведение $[x, y]=(J x, y)$, где $J$ - самосопряженный оператор, такой, что $J^{2}=1$. Здесь и далее в операторных равенствах 1 означает тождественный оператор. Пространство $\mathscr{H}$ всегда считается сепарабельным. Оператор $J$ называют канонической или внутренней симметрией в $\mathscr{H}$. При изучении гамильтоновых систем роль $J$ играет симплектическая матрица. Поскольку оператор $J$ самосопряженный и унитарный одновременно, то его спектр состоит из двух точек \pm 1 . Поэтому справедливо представление $J=P_{+}-P_{-}$, где $P_{+}$и $P_{-}$- взаимно ортогональные ортопроекторы, причем $P_{+}+P_{-}=1$. Пространство $(\mathscr{H},[\cdot, \cdot])$ называют пространством Понтрягина и обозначают через $\Pi_{\varkappa}$, если одно из чисел $\operatorname{rank} P_{+}$или $\operatorname{rank} P_{-}$конечно и равно $\varkappa$, и пространством Крейна, если оба эти числа бесконечны. Подпространство $\mathscr{L}$ в $(\mathscr{H},[\cdot, \cdot])$ называют неотрицательным, если для всех $x \in \mathscr{L}$ выполнено неравенство $[x, x] \geqslant 0$. Если неотрицательное подпространство $\mathscr{L}$ не допускает неотрицательных нетривиальных расширений, то оно называется максимальным. Аналогично определяются максимальные неположительные подпространства в $(\mathscr{H},[\cdot, \cdot])$.

Пусть $A$-линейный оператор в $\mathscr{H}$ с областью определения $\mathscr{D}(A)$. Через $\sigma(A)$ и $\rho(A)$ обозначаем его спектр и резольвентное множество соответственно. Если оператор $A$ замыкаем, то через $\bar{A}$ обозначаем его замыкание. Плотно определенный оператор $A$ называется диссипативным в $\mathscr{H}$, если $\operatorname{Re}(A x, x) \leqslant 0$ для всех $x \in \mathscr{D}(A)$. Диссипативный оператор называется максимально диссипативнымм или $m$-диссипативным, если он не допускает нетривиальных диссипативных расширений. Известно [14, гл. V, § 3.10], что последнее условие для диссипативного оператора $A$ эквивалентно условию $\rho(A) \supset \mathbb{C}^{+}$, где $\mathbb{C}^{+}$- открытая правая комплексная полуплоскость. Оператор $A$ называется $J$-диссипативным (J-максимально диссипативным) или диссипативным (m-диссипативным) в пространстве $(\mathscr{H},[\cdot, \cdot])$, если $J A$ - диссипативный $(m$-диссипативный) оператор в $\mathscr{H}$. Аналогично, А называется симметрическим (самосопряженным) в

*Работа поддержана программой «Ведущие научные школы», грант № НШ-5247.2006.1, Российским фондом фундаментальных исследований, грант № 07-01-00283, и фондом ИНТАС, грант № 05-1000008-7883. 
пространстве $(\mathscr{H},[\cdot, \cdot])$, если JA симметричен (самосопряжен) в пространстве $\mathscr{H}$.

Представим пространство $\mathscr{H}$ в виде ортогональной суммы, $\mathscr{H}=\mathscr{H}^{+} \oplus \mathscr{H}^{-}$, где $\mathscr{H}^{ \pm}=P_{ \pm} \mathscr{H}$ - образы проекторов $P_{ \pm}$. Пусть $A$ - линейный оператор в $\mathscr{H}$ с областью определения $\mathscr{D}(A)$. Положим $\mathscr{D}^{ \pm}=\mathscr{D}(A) \cap \mathscr{H}^{ \pm}$. Далее мы будем иметь дело только с такими операторами, для которых сумма $\mathscr{D}^{+} \oplus \mathscr{D}^{-}$является существенной областъю $^{1)}$ оператора $A$. Это означает следующее: если $A_{0}-$ сужение оператора $A$ на $\mathscr{D}^{+} \oplus \mathscr{D}^{-}$, то $A \subset \bar{A}_{0}$, где $\bar{A}_{0}$ - замыкание оператора $A$.

Рассматривая, если нужно, сужение оператора $A$, будем далее считать, что $\mathscr{D}(A)=\mathscr{D}^{+} \oplus \mathscr{D}^{-}$. В этом случае $A$ допускает представление в виде операторной матрицы по отношению к каноническому разложению $\mathscr{H}=\mathscr{H}^{+} \oplus \mathscr{H}^{-}$:

$$
A=\left(\begin{array}{ll}
P_{+} A P_{+} & P_{+} A P_{-} \\
P_{-} A P_{+} & P_{-} A P_{-}
\end{array}\right):=\left(\begin{array}{ll}
A_{11} & A_{12} \\
A_{21} & A_{22}
\end{array}\right) .
$$

При таком представлении векторы $x=x_{+}+x_{-} \in \mathscr{H}$, где $x_{ \pm} \in \mathscr{H}^{ \pm}$, отождествляются со столбцами $x=\left(\begin{array}{l}x_{+} \\ x_{-}\end{array}\right)$и действие $A$ задается равенством

$$
A x=A\left(\begin{array}{l}
x_{+} \\
x_{-}
\end{array}\right)=\left(\begin{array}{c}
A_{11} x_{+}+A_{12} x_{-} \\
A_{21} x_{+}+A_{22} x_{-}
\end{array}\right), \quad x_{+} \in \mathscr{D}^{+}, x_{-} \in \mathscr{D}^{-} .
$$

В 1941 г. С. Л. Соболев обратил внимание Л. С. Понтрягина на следующий замечательный факт: самосопряженный оператор в пространстве $\Pi_{1}$ всегда имеет по менъшей мере один собственный вектор (доказательство Соболева долгое время оставалось неопубликованным; этот результат Соболев применял для исследования устойчивости одной задачи механики [28]). Это наблюдение Соболева послужило толчком для исследования Понтрягина [24], где он заложил начала геометрии пространств с индефинитной метрикой и получил следующий фундаментальный результат.

Теорема Понтрягина. Пусть $A$ - самосопряженный оператор в $\mathrm{npocm-}$ ранстве $\Pi_{\varkappa}$, причем $\operatorname{rank} P_{+}=\varkappa<\infty$. Тогда существует $A$-инвариантное максимальное неотрицательное подпространство $\mathscr{L}(\operatorname{dim} \mathscr{L}=\varkappa)$, такое, ито спектр сужения $\left.A\right|_{\mathscr{L}}$ лежит в замкнутой верхней полуплоскости.

После работы [24] проблема существования инвариантных максимальных полудефинитных подпространств оказалась в центре внимания теории операторов в пространствах Понтрягина и Крейна. Крейн [16] получил аналог теоремы Понтрягина для унитарных операторов в $\Pi_{\varkappa}$, развивая другой подход к проблеме, нежели в [24]. Важное обобщение теоремы Понтрягина было получено Лангером [19], [20] и Крейном [17]. Сформулируем результат из [20].

Теорема Лангера. Пусть $A$-самосопряэсенный оператор в пространстве Крейна $(\mathscr{H},[\cdot, \cdot])$, причем $\mathscr{D}(A) \supset \mathscr{H}^{+}$(это условие эквивалентно возмоэнности представления (1.1), в котором операторы $A_{11}$ и $A_{21}$ ограничены). Если оператор $A_{12}=P_{+} A P_{-}$компактен, то существует $A$-инвариантное максимальное неотрицательное подпространство $\mathscr{L}$, такое, что спектр суэсения $\left.A\right|_{\mathscr{L}}$ лежит в замкнутой верхней полуплоскости.

1) В английском языке для этого понятия резервируется слово «соre». В русском издании монографии [14] это слово не совсем удачно переведено как «ядро». 
Впоследствии теоремы о существовании $A$-инвариантных подпространств были получены для других классов операторов. Крейн ввел и исследовал класс дефинитных операторов, а позже для более широкого класса так называемых дефинизируемых операторов Лангер [21], [22] доказал теорему о существовании максимальных неотрицательных и неположительных инвариантных подпространств, а также установил существование спектральной функции для таких операторов. Эти исследования получили развитие в работах Йонаса [12], [13] и Дритшеля [6], [7]. Крейн и Лангер [18] и независимо Азизов [2], [3] начали исследование диссипативных операторов в пространствах Понтрягина и Крейна. В частности, в этих работах они доказали следующий результат.

Теорема Крейна-Лангера-Азизова. Пусть -iA-максимально диссипативный оператор в пространстве Понтрягина $\Pi_{\varkappa}$. Тогда справедливо утверждение теоремы Понтрягина.

Далее, Азизов и Хорошавин [4] доказали аналог теоремы Лангера для некоторого класса нерастягивающих операторов в пространстве Крейна, а с помощью этого результата Азизов [5, гл. 2] доказал, что теорема Лангера из [20] остается справедливой для максимально диссипативных операторов в пространстве Крейна (при этом в формулировке верхнюю комплексную полуплоскость нужно заменить на левую). Более того, Азизов показал, что условие компактности оператора $A_{12}$ можно заменить на условие его компактности по отношению к оператору $A_{22}$, т. е. компактности оператора $A_{12}\left(A_{22}-\mu\right)^{-1}$ при некотором $\mu \in \rho\left(A_{22}\right)$ (для самосопряженных операторов этот факт ранее был отмечен Крейном [17]). Прямое, более короткое доказательство теоремы Азизова было предложено автором [25].

Задачу описания вещественного спектра или чисто мнимого спектра для сужений самосопряженных или диссипативных операторов в пространстве Крейна на максимальное неотрицательное инвариантное подпространство изучали Костюченко и Оразов [15], Гомилко [10] и автор [26].

Условие Лангера $\mathscr{D}(A) \supset \mathscr{H}^{+}$(или условие ограниченности в матричном представлении (1.1) операторов $A_{11}$ и $\left.A_{21}\right)$ является весьма ограничительным. В частности, оно не выполняется в некоторых конкретных задачах, которые были модельными в работах [1], [23]). Это обстоятельство послужило мотивом для получения обобщений сформулированных результатов, когда условие Лангера заменяется другим, более слабым. Такой результат был недавно получен автором [27]. Условие Лангера в [27] заменено следующим условием: найдется число $\mu$ в левой полуплоскости, такое, что операторы

$$
\left(A_{22}-\mu\right)^{-1} A_{21}, \quad A_{12}\left(A_{22}-\mu\right)^{-1}, \quad S(\mu)=A_{11}-A_{12}\left(A_{22}-\mu\right)^{-1} A_{21}
$$

ограничены. Оператор-функцию $S(\mu)$ называют передаточной функиией (а также трансфер-функцией или дополнением Фробениуса-Шура). Язык, использующий передаточные функции, на первый взгляд может показаться неуклюжим, но он естествен, и соответствующие условия в конкретных задачах нетрудно проверять. При этом условие ограниченности первых двух операторов, как правило, выполняется автоматически. Но условие ограниченности передаточной функции является достаточно «жестким». Ниже мы увидим, что оно выполняется в том и только том случае, когда сужение оператора $A$ на соответствующее инвариантное подпространство является ограниченным оператором. Конечно, 
имея в виду дальнейшие приложения (которые в этой работе мы не рассматриваем), представляется привлекательной задача от этого условия избавиться. Это является основной иелъю настоящей работы. Но научившись находить инвариантные подпространства, такие, что сужения $\left.A\right|_{\mathscr{L}}$ неограниченны, мы приходим к новой важной задаче: являются ли они генераторами полугрупп какого-либо типа? Найти достаточные условия для положительного ответа на этот вопрос - вторая иель работы.

\section{§2. Существование инвариантных подпространств}

В случае неограниченных операторов важно определить, в каком смысле понимается инвариантность подпространств. Мы сделаем это в случае подпространств специального вида

$$
\mathscr{L}=\left\{x=\left(\begin{array}{c}
x_{+} \\
K x_{+}
\end{array}\right), x_{+} \in \mathscr{H}^{+}\right\},
$$

где $K: \mathscr{H}^{+} \rightarrow \mathscr{H}^{-}$- линейный ограниченный оператор. О таких подпространствах говорят, что они имеют графб-представление, или называют их граф-подпространствами, а $K$ - угловым оператором. Подпространство $\mathscr{L}$ такого вида будем называть $A$-инвариантным, если из условия $x_{+} \in \mathscr{D}(A)$ следуют включения $K x_{+} \in \mathscr{D}(A)$ и $A x \in \mathscr{L}$ для всех $x \in \mathscr{D}(A) \cap \mathscr{L}$. Легко проверить, что если граф-подпространство $\mathscr{L}$ является $A$-инвариантным в смысле приведенного определения и $A$ замыкаем, то $\mathscr{L}$ инвариантно в смысле того же определения относительно замыкания $\bar{A}$ этого оператора. Приведенное определение согласуется с пониманием инвариантности в предшествующих работах: при выполнении условия Лангера $\mathscr{H}^{+} \subset \mathscr{D}(A)$ подпространство $\mathscr{L}$ считалось инвариантным, если $\mathscr{L} \subset \mathscr{D}(A)$ и $A(\mathscr{L}) \subset \mathscr{L}$.

Далее будет использоваться следующий хорошо известный факт (см. [24], [5]): подпространство $\mathscr{L}$ является максимальным неотрицательным, если и только если оно является граф-подпространством с нерастягивающим угловым оператором (m.e. $\|K\| \leqslant 1)$.

Основная теорема об инвариантных подпространствах будет доказана для $m$-диссипативных операторов в пространстве Крейна. Известно [5, теорема II.2.9], что при выполнении условия Лангера $\mathscr{D}(A) \supset \mathscr{H}^{+}$оператор $A m$-диссипативен в $(\mathscr{H},[\cdot, \cdot])$ тогда и только тогда, когда $-A_{22}$ максимально диссипативен в $\mathscr{H}^{-}$, т. е. левая полуплоскость лежит в резольвентном множестве оператора $A_{22}$. В общем случае можно утверждать, что из $m$-диссипативности оператора $A$ вытекает только диссипативность операторов $A_{11}$ и $-A_{22}$. Но мы будем работать с передаточной функцией, поэтому будем требовать выполнения только условия существования резольвенты оператора $A_{22}$ при некотором (а тогда при всех) $\mu \in \mathbb{C}^{-}$. Сущность налагаемых ниже условий состоит в том, что оператор $A_{22}$ является доминирующим по отношению к сплетающим операторам $A_{21}$ и $A_{12}$. Сформулируем основной результат.

Теорема 2.1. Пусть $A$ - диссипативный оператор в пространстве Крейна $(\mathscr{H},[\cdot, \cdot])$ с областью определения $\mathscr{D}(A)=\mathscr{D}^{+} \oplus \mathscr{D}^{-}$, плотной в $\mathscr{H}=$ $\mathscr{H}^{+} \oplus \mathscr{H}^{-}$. Пусть $(1.1)$ - его матричное представление в $\mathscr{H}^{+} \oplus \mathscr{H}^{-}$, и пусть выполнены следующие условия:

(i) оператор $-A_{22}$ является $m$-диссипативным в пространстве $\mathscr{H}^{-}$(т.е. существует резольвента $\left(A_{22}-\mu\right)^{-1}$ при всех $\left.\mu \in \mathbb{C}^{-}\right)$; 
(ii) оператор $F(\mu)=\left(A_{22}-\mu\right)^{-1} A_{21}$ допускает ограниченное замъкание при некотором (а тогда при всех) $\mu \in \mathbb{C}^{-}$;

(iii) оператор $G(\mu)=A_{12}\left(A_{22}-\mu\right)^{-1}$ компактен при некотором (а тогда при всех) $\mu \in \mathbb{C}^{-}$.

Тогда существует максимальное неотрицательное подпространство, инвариантное относительно оператора $A . B$ случае когда замыкание $\bar{A}$ является m-диссипативным в $(\mathscr{H},[\cdot, \cdot])$ (и только в этом случае), найдется максимальное неотрицательное $A$-инвариантное подпространство $\mathscr{L}^{+}$, такое, что спектр сужения $A_{+}=\left.\bar{A}\right|_{\mathscr{L}^{+}}$содержится в замкнутой левой полуплоскости.

Сразу отметим, что в условиях теоремы не нужно дополнительно требовать замыкаемости оператора $A$. Всякий плотно определенный диссипативный оператор в пространстве Крейна замыкаем (см., например, [5, гл. I, §2]). Доказательству теоремы предпошлем ряд лемм. Первая из них известна и доказана в [1]. Основную роль в дальнейшем играют леммы 2.3, 2.4 и 2.8. Далее, там, где не возникает недоразумений, мы будем опускать указание на зависимость операторов $G(\mu), F(\mu), S(\mu)$ от выбора $\mu \in \mathbb{C}^{-}$и писать $G, F, S$.

Лемма 2.2. Пусть $A$ - оператор с плотной областью определения $\mathscr{D}(A)=$ $\mathscr{D}^{+} \oplus \mathscr{D}^{-}$, резольвентное множество $\rho\left(A_{22}\right)$ непусто, а операторъ

$$
G=A_{12}\left(A_{22}-\mu\right)^{-1} \quad u \quad F=\left(A_{22}-\mu\right)^{-1} A_{21}, \quad \mu \in \rho\left(A_{22}\right),
$$

ограничены при некотором (а тогда при всех) $\mu \in \rho\left(A_{22}\right)$. Тогда оператор $A$ замыкаем в том и только том случае, когда $S=A_{11}-A_{12} F$ замыкаем в $\mathscr{H}^{+}$, и замыкание $\bar{A}$ определяется равенством

$$
\bar{A}=\mu+\left(\begin{array}{cc}
1 & G \\
0 & 1
\end{array}\right)\left(\begin{array}{cc}
\bar{S}-\mu & 0 \\
0 & A_{22}-\mu
\end{array}\right)\left(\begin{array}{cc}
1 & 0 \\
\bar{F} & 1
\end{array}\right) .
$$

Более точно, область определения и действие оператора $\bar{A}$ определяются равенствами

$$
\begin{aligned}
\mathscr{D}(\bar{A})= & \left\{\left(\begin{array}{l}
x_{+} \\
x_{-}
\end{array}\right) \in \mathscr{H}, x_{+} \in \mathscr{D}(\bar{S}), \bar{F} x_{+}+x_{-} \in \mathscr{D}^{-} \subset \mathscr{D}\left(A_{22}\right)\right\}, \\
& \bar{A}\left(\begin{array}{l}
x_{+} \\
x_{-}
\end{array}\right)=\left(\begin{array}{c}
\bar{S} x_{+}+G\left(A_{22}-\mu\right)\left(\bar{F} x_{+}+x_{-}\right) \\
\left(A_{22}-\mu\right)\left(\bar{F} x_{+}+x_{-}\right)+\mu x_{-}
\end{array}\right) .
\end{aligned}
$$

Лемма 2.3. При $\mu \in \rho\left(A_{22}\right)$ справедливо равенство

$$
J A+\mu=J\left(\begin{array}{cc}
1 & G \\
0 & 1
\end{array}\right)\left(\begin{array}{cc}
S+\mu & 0 \\
0 & A_{22}-\mu
\end{array}\right)\left(\begin{array}{cc}
1 & 0 \\
F & 1
\end{array}\right), \quad J:=\left(\begin{array}{cc}
1 & 0 \\
0 & -1
\end{array}\right) .
$$

Доказательство. В справедливости этого равенства легко убедиться непосредственной проверкой с учетом представления (2.2), в котором вместо замыканий $\bar{A}, \bar{F}$ и $\bar{S}$ участвуют $A, F$ и $S$. Конечно, равенство (2.3) остается верным, если в нем операторы $A, F, S$ заменить на их замыкания.

Лемма 2.4. При $\mu \in \rho\left(A_{22}\right)$ для всех $x_{+} \in \mathscr{D}_{+}$справедливо равенство

$$
\left(S x_{+}, x_{+}\right)=\left(J A\left(\begin{array}{c}
x_{+} \\
-F x_{+}
\end{array}\right),\left(\begin{array}{c}
x_{+} \\
-F x_{+}
\end{array}\right)\right)+\mu\left(F x_{+}, F x_{+}\right) .
$$

$B$ частности, при любом $\mu \in \mathbb{C}^{-}$оператор $S=S(\mu)$ с областью определения $\mathscr{D}(S)=\mathscr{D}^{+}$является диссипативным в пространстве $\mathscr{H}^{+}$, если оператор 
A диссипативен в пространстве $(\mathscr{H},[\cdot, \cdot])$. Оператор $\bar{S}$ является $m$-диссипативным в пространстве $\mathscr{H}^{+}$, если $\bar{A}$ является $m$-диссипативным в пространстве $(\mathscr{H},[\cdot, \cdot])$, и наоборот.

Доказательство. В справедливости равенства (2.4) легко убедиться непосредственной проверкой с помощью равенства (2.3). Эквивалентность $m$-диссипативности операторов $\bar{S}$ в пространстве $\mathscr{H}^{+}$и $J \bar{A}$ в пространстве $\mathscr{H}$ также следует из равенства (2.3).

Лемма 2.5. Пусть подпространство $\mathscr{L}$ имеет представление (2.1), m.е. является граф-подпространством. Тогда $\mathscr{L}$ является $A$-инвариантным в том и только том случае, когда выполняется равенство

$$
(1-K G)\left(A_{22}-\mu\right)(F+K)=K(S-\mu) .
$$

Здесь предполагается, что областью определения оператора в правой ( а потому и в левой) части равенства является линеал $\mathscr{D}(S)=\mathscr{D}^{+}$.

Доказательство. Пусть подпространство $\mathscr{L}$ является $A$-инвариантным. Тогда $K x_{+} \in \mathscr{D}^{-}$для всех $x_{+} \in \mathscr{D}^{+}$, и из равенства $(2.2)$ находим

$$
(A-\mu)\left(\begin{array}{c}
x_{+} \\
K x_{+}
\end{array}\right)=\left(\begin{array}{c}
(S-\mu) x_{+}+G\left(A_{22}-\mu\right)(F+K) x_{+} \\
\left(A_{22}-\mu\right)(F+K) x_{+}
\end{array}\right) .
$$

Здесь правая часть принадлежит $\mathscr{L}$; поэтому, обозначая первую координату через $y_{+}$, получаем

$$
\left[(S-\mu)+G\left(A_{22}-\mu\right)(F+K)\right] x_{+}=y_{+}, \quad\left(A_{22}-\mu\right)(F+K) x_{+}=K y_{+} .
$$

Подставляя выражение для $y_{+}$во второе равенство, приходим к уравнению (2.5).

Обратно, если выполнено уравнение (2.5), то при всех $x_{+} \in \mathscr{D}^{+}$справедливы два последних уравнения. Тогда $\left(A_{22}-\mu\right) K x_{+}=K y_{+}-A_{21} x_{+}$, а следовательно, $K x_{+} \in \mathscr{D}^{-}$. Поэтому

$$
\left(\begin{array}{c}
x_{+} \\
K x_{+}
\end{array}\right) \in \mathscr{D}(A) \quad \text { и } \quad A\left(\begin{array}{c}
x_{+} \\
K x_{+}
\end{array}\right) \in \mathscr{L} \quad \text { для любого } x \in \mathscr{D}^{+},
$$

т.е. $\mathscr{L}$ является $A$-инвариантным. Переходя в $(2.5)$ к замыканиям и вспоминая описание области $\mathscr{D}(\bar{A})$, непосредственно получаем, что $\mathscr{L}$ является также $\bar{A}$-инвариантным.

Замечание 2.6. Ранее, начиная с работы Понтрягина [24], использовалась другая версия леммы 2.5: $\mathscr{L}$ является $A$-инвариантным тогда и только тогда, когда

$$
A_{21}+A_{22} K-K A_{11}-K A_{12} K=0 .
$$

Это уравнение называют уравнением Риккати, ассоциированным с операторматрицей $A$. Однако в случае, когда все элементы оператор-матрицы $A$ могут быть неограниченными операторами, удобнее иметь дело с модифицированным уравнением Риккати (2.5).

Лемма 2.7. Пусть $\mathscr{H}_{1}, \mathscr{H}_{2}$ - гилъбертовы пространства, оператор -T: $\mathscr{H}_{1}$ $\rightarrow \mathscr{H}_{1}$ является m-диссипативным, а В: $\mathscr{H}_{1} \rightarrow \mathscr{H}_{2}$ Т-компактен. Тогда

$$
\left\|B(T-\mu)^{-1}\right\| \rightarrow 0 \quad \text { nрu } \mu \rightarrow \infty
$$

равномерно в любом секторе $\Lambda_{\gamma}=\{\lambda:|\arg (\pi-\mu)| \leqslant \gamma\}, \gamma<\pi / 2$. 
Доказательство. Положим $G(\mu)=B(T-\mu)^{-1}$. По условию $G(\mu)$ компактен при некотором (а тогда при всех) $\mu \in \mathbb{C}^{-}$. Воспользуемся равенством

$$
G(\mu)=G(-1)(T+1)(T-\mu)^{-1} .
$$

Очевидно, что в условиях леммы норма оператор-функции $(T+1)(T-\mu)^{-1}$ равномерно ограничена в секторе $\Lambda_{\gamma}$ вне малой окрестности нуля. Компактный оператор $G(-1)$ можно приблизить с произвольной точностью в операторной норме конечномерным оператором. Поэтому достаточно показать, что $\left\|V(T+1)(T-\mu)^{-1}\right\| \rightarrow 0$ при $\mu \rightarrow \infty$ в левой полуплоскости для любого одномерного оператора $V=(\cdot, \varphi) \psi$, где $\varphi \in \mathscr{H}_{1}, \psi \in \mathscr{H}_{2}$. Заметим, что $V$ можно с произвольной точностью приблизить в операторной норме оператором $V_{0}=$ $\left(\cdot, \varphi_{0}\right) \psi$, где $\varphi_{0} \in \mathscr{D}\left(T^{*}\right)$ (в условиях леммы сопряженный оператор $T^{*}$ плотно определен). Но оператор $V_{0}(T+1)$ ограничен, a $\left\|(T-\mu)^{-1}\right\| \leqslant(\cos \gamma)^{-1}|\mu|^{-1}$ в секторе $\Lambda_{\gamma}$. Отсюда получаем утверждение леммы.

В силу леммы 2.7 найдутся значения $\mu \in \mathbb{C}^{-}$, для которых $\|G(\mu)\|<1 / 2$, т. е. множество $\mu \in \mathbb{C}^{-} \cap \rho(1-K G)$ непусто, если $\|K\| \leqslant 1$. Тогда из леммы 2.5 получаем следующий важный для дальнейшего результат.

Лемма 2.8. Пусть число $\mu \in \mathbb{C}^{-}$maкое, что $\|G(\mu)\|<1 / 2$. Тогда оператор А обладает инвариантным максимальным неотрищательным подпространством в том и только том случае, когда найдется нерастягивающий оператор $K: \mathscr{H}^{+} \rightarrow \mathscr{H}^{-}$, такой, что

$$
F+K=\left(A_{22}-\mu\right)^{-1}(1-K G)^{-1} K(S-\mu), \quad \mathscr{D}(S)=\mathscr{D}^{+} .
$$

Решение $K$ этого уравнения не зависит от $\mu \in \mathbb{C}^{-} \cap \rho(1-K G)$.

Доказательство. Первое утверждение леммы непосредственно следует из леммы 2.5. Далее, уравнение (2.7) эквивалентно уравнению (2.5), где операторы в обеих частях равенств определены на $\mathscr{D}^{+}$. Поскольку операторы $A_{21}=$ $\left(A_{22}-\mu\right) F$ и $K A_{11}$ корректно определены на $\mathscr{D}^{+}$, то $\left(A_{22}-\mu\right) K$ и $K A_{12} F$ обладают тем же свойством. Но тогда в (2.5) можно раскрыть скобки и получить уравнение (2.6), в котором все слагаемые корректно определены на $\mathscr{D}^{+}$. Легко также убедиться в справедливости обратного утверждения: если уравнение (2.6) выполнено на линеале $\mathscr{D}^{+}$, то при любом $\mu \in \mathbb{C}^{-}$выполняется уравнение $(2.5)$ на той же области определения (нужно проделать обратные преобразования). Таким образом, $K$ не зависит от $\mu$.

Напомним [9, гл. 6], что система $\left\{e_{k}\right\}_{k=1}^{\infty}$ элементов гильбертова пространства $\mathscr{H}$ называется безусловным базисом, если любой элемент $x \in \mathscr{H}$ однозначно представим в виде ряда $\sum c_{k} e_{k}$, безусловно сходящегося по норме пространства $\mathscr{H}$. Числовые коэффициенты $c_{k}=c_{k}(x)$ в этом представлении являются линейными непрерывными функционалами; поэтому $c_{k}(x)=\left(x, e_{k}^{*}\right)$. Систему $\left\{e_{k}^{*}\right\}_{k=1}^{\infty}$ называют сопряжсенной; она тоже является безусловным базисом. Если безусловный базис почти нормирован (т. е. $c_{1}<\left\|e_{k}\right\|<c_{2}$ при некоторых $\left.c_{1}, c_{2}>0\right)$, то его называют базисом Рисса.

Лемма 2.9. Пусть $T$ - плотно определенный замыкаемый оператор в сепарабельном гильбертовом пространстве $\mathscr{H}$ и $\mathscr{H}_{T}$ - плотно вложенное в $\mathscr{H}$ гильбертово пространство, снабжсенное нормой графика

$$
\|x\|_{T}=\sqrt{\|\bar{T} x\|^{2}+\|x\|^{2}} .
$$


Существует система $\left\{e_{k}\right\}_{k=1}^{\infty}, e_{k} \in \mathscr{D}(T)$, являющаяся безусловньм базисом в обоих пространствах $\mathscr{H} u \mathscr{H}_{T}$. В частности, если $\left\{e_{k}^{*}\right\}_{k=1}^{\infty}-$ сопряэсенная $\kappa$ ней система, то проекторь $P_{n}=\sum_{1}^{n}\left(\cdot, e_{k}^{*}\right) e_{k}$ на подпространства $\mathscr{L}_{n}=$ $\operatorname{Lin}\left\{e_{k}\right\}_{k=1}^{n}$ (а также сопряэсенные $\kappa$ ним проекторы $\left.P_{n}^{*}\right)$ сильно сходятся $\kappa$ тожсдественному оператору в обоих пространствах $\mathscr{H}$ u $\mathscr{H}_{T}$.

Доказательство. Сначала построим систему $\left\{\varphi_{k}\right\}_{k=1}^{\infty}, \varphi_{k} \in \mathscr{D}(\bar{T})$, которая является ортонормированным базисом в $\mathscr{H}$ и безусловным базисом в $\mathscr{H}_{T}$.

Известно [14, теорема V.3.24], что оператор $T^{*} \bar{T}$ самосопряжен в $\mathscr{H}$, а его область определения $\mathscr{D}\left(T^{*} \bar{T}\right)$ является существенной областью для оператора $\bar{T}$ и потому плотна в $\mathscr{H}_{T}=\mathscr{D}(\bar{T})$. Из определения нормы в пространстве $\mathscr{H}_{T}$

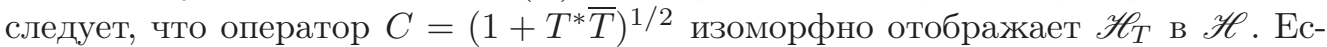
ли вложение $\mathscr{H}_{T} \hookrightarrow \mathscr{H}$ компактно, то оператор $C^{-1}: \mathscr{H} \rightarrow \mathscr{H}$ компактный. В качестве искомой системы тогда можно взять полную ортонормированную в $\mathscr{H}$ систему $\left\{\psi_{k}\right\}_{1}^{\infty}$ собственных векторов этого оператора, поскольку система $\left\{\mu_{k} \psi_{k}\right\}_{1}^{\infty}$, где $\left\{\mu_{k}\right\}_{1}^{\infty}$ - последовательность собственных значений оператора $C^{-1}$, будет полной и ортонормированной в пространстве $\mathscr{H}_{T}$.

Если $C^{-1}: \mathscr{H} \rightarrow \mathscr{H}$ некомпактен, но его спектр состоит только из собственных значений, то доказательство сохраняется: нужно выбрать ортонормированную систему в каждом собственном подпространстве и взять объединение этих систем. В общем случае рассмотрим оператор $C_{1}=1+\sum_{k=1}^{\infty} k\left(E_{k}-E_{k-1}\right)$, где $E_{\lambda}$ - спектральная функция оператора $C$. Очевидно, что спектр оператора $C_{1}$ состоит только из собственных значений, причем операторы $C^{-1} C_{1}$ и $C_{1}^{-1} C$ ограничены, т. е. пространства $\mathscr{H}_{T}=\mathscr{H}_{C}$ и $\mathscr{H}_{C_{1}}$ совпадают и нормы в них эквивалентны. Поэтому, если взять систему с требуемыми свойствами для оператора $C_{1}$, то она будет безусловным базисом в $\mathscr{H}_{T}$.

Итак, построена ортонормированная в $\mathscr{H}$ система $\left\{\varphi_{k}\right\}_{k=1}^{\infty}, \varphi_{k} \in \mathscr{D}(\bar{T})$, являющаяся безусловным базисом в $\mathscr{H}_{T}$. Из определения замыкания $\bar{T}$ следует, что линеал $\mathscr{D}(T)$ плотен в $\mathscr{H}_{T}$. Положим $\alpha_{k}=\left\|\varphi_{k}\right\|_{T}$ и выберем элементы $e_{k} \in \mathscr{D}(T)$ так, чтобы

$$
\sum \alpha_{k}^{2}\left\|\alpha_{k}^{-1} \varphi_{k}-e_{k}\right\|_{T}^{2}<\delta
$$

Тогда в силу неравенств $\|x\|_{T} \geqslant\|x\|, \alpha_{k} \geqslant 1$

$$
\sum\left\|\alpha_{k}^{-1} \varphi_{k}-e_{k}\right\|_{T}^{2}<\delta, \quad \sum\left\|\varphi_{k}-\alpha_{k} e_{k}\right\|^{2}<\delta .
$$

Поскольку система $\left\{\alpha_{k}^{-1} \varphi_{k}\right\}-$ базис Рисса в $\mathscr{H}_{T}$, то, согласно теореме Бари [9, гл. 6], система $\left\{e_{k}\right\}_{k=1}^{\infty}$ обладает тем же свойством, если число $\delta<1$ выбрано достаточно малым. Так как $\left\{\varphi_{k}\right\}$ - ортонормированная система в $\mathscr{H}$, то в силу той же теоремы Бари система $\left\{\alpha_{k} e_{k}\right\}$ является базисом Рисса в пространстве $\mathscr{H}$ при любом $\delta<1$. Лемма доказана.

Лемма 2.10. Пусть $\mathscr{H}_{1}$ и $\mathscr{H}_{2}$ - гильбертовы пространства и последовательность операторов $K_{n}: \mathscr{H}_{1} \rightarrow \mathscr{H}_{2}$ слабо сходится $\kappa$ оператору $K: \mathscr{H}_{1} \rightarrow$ $\mathscr{H}_{2}$ при $n \rightarrow \infty$ (будем писать $\left.K_{n} \rightarrow K\right)$. Пусть последовательности операторов $B_{n}: \mathscr{H}_{2} \rightarrow \mathscr{H}_{1}$ и $D_{n}: \mathscr{H}_{1} \rightarrow \mathscr{H}_{2}$ сильно сходятся $\kappa$ В и $D$ соответственно $\left(\right.$ пишем $\left.B_{n} \rightarrow B, D_{n} \rightarrow D\right)$. Тогда

$$
K_{n} D_{n} \rightarrow K D, \quad B_{n} K_{n} \rightarrow B K, \quad B_{n} K_{n} D_{n} \rightarrow B K D .
$$


Если $G: \mathscr{H}_{2} \rightarrow \mathscr{H}_{1}$ - компактный оператор, а оператор $U: \mathscr{H}_{1} \rightarrow \mathscr{H}_{1}$ ограничен, то при любом $s=1,2, \ldots$

$$
\left(G K_{n}\right)^{s} \rightarrow(G K)^{s}, \quad\left(K_{n} G\right)^{s} K_{n}=K_{n}\left(G K_{n}\right)^{s} \rightarrow(K G)^{s} K, \quad G K_{n} U G \Rightarrow G K U G,
$$

где знак $\Rightarrow$ означает сходимость в равномерной операторной топологии.

Доказательство. Конечно, сформулированные утверждения в разных формах отмечались и использовались во многих книгах и работах. Похожие утверждения можно найти, например, в [11, гл. 12].

Доказательства легче провести независимо. Например, из определения слабой сходимости при любых $x \in \mathscr{H}_{1}, y \in \mathscr{H}_{2}$ имеем

$$
\left|\left(K_{n} D_{n} x-K D x, y\right)\right|=\left|\left(D_{n} x-D x, K_{n}^{*} y\right)+\left(\left(K_{n}-K\right) D x, y\right)\right| \rightarrow 0 \quad \text { при } n \rightarrow \infty \text {. }
$$

Из этого следует первое утверждение в (2.8). Второе получается аналогично, а третье следует из первых двух. Так как компактный оператор $G$ можно с произвольной точностью приблизить конечномерным оператором, то утверждения (2.9) достаточно доказать для одномерного оператора $G=(\cdot, \varphi) \psi$, где $\varphi \in \mathscr{H}_{2}$, $\psi \in \mathscr{H}_{1}$, причем достаточно проверить первое и третье утверждения. Но в этом случае проверка тривиальна.

Лемма 2.11. Пусть $\mathscr{H}_{1}, \mathscr{H}_{2}$ - гильбертовы пространства, G: $\mathscr{H}_{2} \rightarrow \mathscr{H}_{1}$ - компактный оператор, оператор $T: \mathscr{H}_{1} \rightarrow \mathscr{H}_{1}$ плотно определен и замкнут, a $B: \mathscr{H}_{1} \rightarrow \mathscr{H}_{1}$ является T-подчиненным, т.е. при некоторых $a, b>0$ справедлива оценка

$$
\|B x\| \leqslant b\|T x\|+a\|x\| \quad \text { для любого } x \in \mathscr{D}(T) \subset \mathscr{D}(B) .
$$

Пусть последовательность операторов $D_{n}: \mathscr{H}_{1} \rightarrow \mathscr{H}_{2}$ слабо сходится $к$ оператору $D$, а последовательность $E_{n}: \mathscr{H}_{1} \rightarrow \mathscr{H}_{1}$ сходится $\kappa$ нулю в равномерной операторной топологии. Пусть область $\Omega \subset \mathbb{C}$ лежит в резольвентном множестве оператора $T$ и $\Omega \cap \rho(T+G D B)$ непусто.

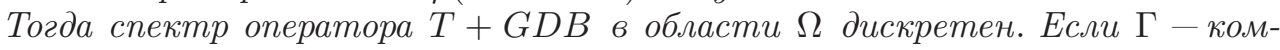
пакт в $\Omega$, не содержащий собственных значений этого оператора, то при достаточно больиих $n$ операторы $T+G D_{n} B+E_{n}-\lambda$ обратимь при $\lambda \in \Gamma u$ равномерно на Г имеет место сильная резольвентная сходимость

$$
\left(T+G D_{n} B+E_{n}-\lambda\right)^{-1} \rightarrow(T+G D B-\lambda)^{-1} \text { nрu } n \rightarrow \infty .
$$

$B$ частности, если при всех достаточно больиих $n>n_{0}$ в $\Omega$ не содержится mочек спектра оператора $T+G D_{n} B+E_{n}$, mo $\rho(T+G D B) \supset \Omega$.

Доказательство. Последнее утверждение этой леммы в разных частных случаях и разными методами доказано в [5] (см. доказательство теоремы III.2.8) и в [25, лемма 5]. Здесь мы проведем доказательство, основанное на синтезе идей из [5] и [25].

Сразу отметим, что дискретность спектра оператора $T+G D B$ в области $\Omega$ следует из теоремы Фредгольма для аналитической оператор-функции $1+$ $G D B(T-\lambda)^{-1}$ (см., например, [9, гл. 1]). Здесь мы учитываем, что условия леммы гарантируют ограниченность оператор-функции $B(T-\lambda)^{-1}$ на любом компакте в $\Omega$. Положим

$$
R(\lambda)=(T+G D B-\lambda)^{-1}, \quad Y_{n}(\lambda)=G\left(D_{n}-D\right) B R(\lambda)+E_{n} R(\lambda) .
$$


Тогда $\left(T+G D_{n} B+E_{n}-\lambda\right)^{-1}=R(\lambda)\left(I+Y_{n}(\lambda)\right)^{-1}$. Поэтому для доказательства соотношения (2.11) достаточно доказать сильную сходимость

$$
\left(1+Y_{n}(\lambda)\right)^{-1} \rightarrow 1, \quad \lambda \in \Gamma .
$$

Оператор-функция $B R(\lambda)=\left[B(T-\lambda)^{-1}\right]\left[1+G D B(T-\lambda)^{-1}\right]^{-1}$ равномерно ограничена на $\Gamma$. Поэтому

$$
Y_{n}^{2}(\lambda)=[*] G\left(D_{n}-D\right)[*] G[*]+\widetilde{E}_{n}(\lambda),
$$

где $\widetilde{E}_{n}(\lambda) \Rightarrow 0$, а знаком $[*]$ обозначены равномерно ограниченные операторфункции, не зависящие от $n$. Из леммы 2.10 следует, что $Y_{n}(\lambda) \rightarrow 0, Y_{n}^{2}(\lambda) \Rightarrow 0$. В частности, $\left\|Y_{n}^{2}(\lambda)\right\|<1 / 2$ при достаточно больших $n>n_{0}$; поэтому из равенства $\left(1-Y_{n}^{2}\right)\left(1+Y_{n}\right)^{-1}=1-Y_{n}$ получаем равномерную оценку

$$
\left\|\left(1+Y_{n}(\lambda)\right)^{-1}\right\|<2\left(1+\left\|Y_{n}(\lambda)\right\|\right) \leqslant \text { const, } \quad n>n_{0} .
$$

Следовательно,

$$
\left(1+Y_{n}(\lambda)\right)^{-1}-1=Y_{n}(\lambda)-Y_{n}^{2}(\lambda)\left(1+Y_{n}(\lambda)\right)^{-1} \rightarrow 0 .
$$

Этим завершается доказательство соотношения (2.11).

Для доказательства последнего утверждения леммы возьмем в качестве $\Gamma$ произвольный жорданов контур в $\Omega$ и проинтегрируем резольвенту $\left(T+G D_{n} B+\right.$ $\left.E_{n}-\lambda\right)^{-1}$ вдоль $\Gamma$. Получим проекторы Рисса $Q_{n}(\Gamma)$, которые сильно сходятся к соответствующему проектору Рисса $Q(\Gamma)$ предельного оператора. Если в $\Omega$ не содержится точек спектра оператора $T+G D_{n} B+E_{n}$, то $Q_{n}(\Gamma)=0$, а потому $Q(\Gamma)=0$ для любого контура $\Gamma \in \Omega$. Но тогда оператор $T+G D B$ не имеет собственных значений в $\Omega$.

Лемма 2.12. Пусть $T: \mathscr{H} \rightarrow \mathscr{H}$ - замкнутый плотно определенный оператор, а В является T-подчиненным, т.е. выполнена оченка (2.10), причем $b<1$. Если $\|T x\| \geqslant \gamma\|x\|$ для всех $x \in \mathscr{D}(T)$ и $a<(1-b) \gamma$, то образы операторов $T$ и $T+B$ замкнуты, а размерности их дефектных подпространств (т.е. размерности ядер операторов $\left.T^{*} u(T+B)^{*}\right)$ совпадают.

Доказательство. Это утверждение есть частный случай теоремы IV.5.22 из [14].

Доказательство теоремы 2.1. Обозначим через $\mathscr{H}_{S}^{+}$вложенное в $\mathscr{H}^{+}$ гильбертово пространство с нормой графика оператора $\bar{S}$. Согласно лемме 2.9 , выберем систему элементов $\left\{e_{k}\right\}_{k=1}^{\infty}, e_{k} \in \mathscr{D}^{+}=\mathscr{D}(S)$, являющуюся безусловным базисом в обоих пространствах $\mathscr{H}^{+}$и $\mathscr{H}_{S}^{+}$. Положим

$$
P_{n}:=\sum_{k=1}^{n}\left(\cdot, e_{k}^{*}\right) e_{k}, \quad \mathscr{H}_{n}^{+}:=\operatorname{Lin}\left\{e_{k}^{*}\right\}_{k=1}^{n}=P_{n}^{*}\left(\mathscr{H}^{+}\right),
$$

где $\left\{e_{k}^{*}\right\}$ - сопряженная система. Тогда $P_{n} \rightarrow 1$ в обоих пространствах $\mathscr{H}^{+}$ и $\mathscr{H}_{S}^{+}$. Рассмотрим операторы

$$
A_{n}=\left(\begin{array}{cc}
P_{n}^{*} A_{11} P_{n} & P_{n}^{*} A_{12} \\
A_{21} P_{n} & A_{22}
\end{array}\right),
$$

действующие в пространствах $\mathscr{H}_{n}^{+} \oplus \mathscr{H}^{-}$. Очевидно, что операторы $A_{n}$ являются $m$-диссипативными в пространстве Понтрягина $\Pi_{n}$. Согласно теореме 
Крейна-Лангера-Азизова, операторы $A_{n}$ обладают $n$-мерными максимальными неотрицательными подпространствами $\mathscr{L}_{n} \subset \mathscr{D}\left(A_{n}\right)$. В силу леммы 2.8 найдутся нерастягивающие операторы $K_{n}: \mathscr{H}_{n}^{+} \rightarrow \mathscr{H}^{-}$, такие, что

$$
F_{n}+K_{n}=\left(A_{22}-\mu\right)^{-1}\left(1_{n}-K_{n} G_{n}\right)^{-1} K_{n}\left(S_{n}-\mu 1_{n}\right),
$$

где $F_{n}=F P_{n}, G_{n}=P_{n}^{*} G, S_{n}=P_{n}^{*} S P_{n}$, а $1_{n}$ - тождественный оператор в $\mathscr{H}_{n}$.

Доопределим операторы $K_{n}$ и $1_{n}$ на все пространство $\mathscr{H}^{+}$, полагая их равными нулю на ортогональном дополнении к $\mathscr{H}_{n}^{+}$. Известно (см., например, [11, гл. 2]), что единичный шар в сепарабельном гильбертовом пространстве является слабо компактным. Так как нормы операторов $K_{n}$ не превосходят единицы, то можно выбрать слабо сходящуюся подпоследовательность $K_{n_{j}} \rightarrow K$. Далее для краткости мы опускаем индекс $j$. Конечно, для предельного оператора $K$ также имеем $\|K\| \leqslant 1$. Учитывая, что $F$ - ограниченный оператор, а $G-$ компактный оператор с нормой $\|G\|<1 / 2$, в силу леммы 2.10 получаем

$$
\begin{gathered}
F P_{n} \rightarrow F, \quad\left(K_{n} P_{n}^{*} G\right)^{s} K_{n} \rightarrow(K G)^{s} K, \quad s=1,2, \ldots, \\
\left(1_{n}-K_{n} P_{n}^{*} G\right)^{-1} K_{n} \rightarrow(1-K G)^{-1} K .
\end{gathered}
$$

Так как оператор $\bar{S}: \mathscr{H}_{S}^{+} \rightarrow \mathscr{H}^{+}$ограничен, а $P_{n} \rightarrow 1$ в $\mathscr{H}_{S}^{+}$, то $\left(\bar{S}_{n}-\mu 1_{n}\right) x \rightarrow$ $(\bar{S}-\mu) x$ для всех $x \in \mathscr{D}(\bar{S})$. Вновь пользуясь леммой 2.10 , получаем, что в уравнении (2.13) можно перейти к слабому пределу. Тогда для предельного оператора $K$ получим уравнение $(2.7)$, справедливое на области $\mathscr{D}(\bar{S})$. В силу леммы 2.8 граф-подпространство с угловым оператором $K$ является максимальным неотрицательным и $A$-инвариантным.

Докажем вторую часть теоремы о существовании инвариантного подпространства $\mathscr{L}^{+}$, такого, что спектр сужения $A_{+}=\left.\bar{A}\right|_{\mathscr{L}_{+}}$лежит в замкнутой левой полуплоскости.

Имеем

$$
(\bar{A}-\mu)\left(\begin{array}{c}
x_{+} \\
K x_{+}
\end{array}\right)=\left(\begin{array}{c}
(\bar{S}-\mu+G L) x_{+} \\
L x_{+}
\end{array}\right), \quad L:=\left(A_{22}-\mu\right)(F+K), \quad \mathscr{D}(L)=\mathscr{D}(\bar{S}) .
$$

Определим оператор $Q: \mathscr{L} \rightarrow \mathscr{H}^{+}$равенством

$$
Q\left(\begin{array}{c}
x_{+} \\
K x_{+}
\end{array}\right)=x_{+}, \quad x=\left(\begin{array}{c}
x_{+} \\
K x_{+}
\end{array}\right) \in \mathscr{L} .
$$

Очевидно, что $Q$ ограниченно обратим, причем $\left\|Q^{-1}\right\| \leqslant \sqrt{2}$. Из равенств (2.5) и (2.14) получаем

$$
\left.(\bar{A}-\alpha)\right|_{\mathscr{L}^{+}}=Q^{-1}(\bar{S}-\alpha+G L) Q=Q^{-1}[\bar{S}-\alpha+V(\bar{S}-\mu)] Q, \quad V:=G(1-G K)^{-1} K .
$$

Здесь $V=V(\mu)$, причем в силу леммы 2.7 число $\mu \in \mathbb{C}^{-}$можно выбрать так, что $\|V\|<1 / 3$. Воспользуемся леммой 2.12 , полагая

$$
T=\bar{S}-\alpha, \quad B=V(\bar{S}-\alpha)+(\alpha-\mu) V .
$$

В этом случае $\left\|T x_{+}\right\| \geqslant(\operatorname{Re} \alpha)\left\|x_{+}\right\|$, а $B$ является $T$-подчиненным с постоянными $a=|\alpha-\mu| / 3, b=1 / 3$. Тогда при всех положительных $\alpha>|\alpha-\mu| / 2$ оператор $(\bar{S}-\alpha)+V(\bar{S}-\mu)$ является обратимым в том и только том случае, когда оператор $\bar{S}-\alpha$ обратим, т. е. когда $\bar{S}$ является $m$-диссипативным. Согласно лемме 2.7, это 
эквивалентно $m$-диссипативности оператора $\bar{A}$ в пространстве $(\mathscr{H},[\cdot, \cdot])$. Следовательно, открытая левая полуплоскость может принадлежать резольвентному множеству сужения $\left.\bar{A}\right|_{\mathscr{L}+}$ только в случае, когда оператор $\bar{A}$ является $m$-диссипативным в пространстве $(\mathscr{H},[\cdot, \cdot])$.

Пусть $A$ обладает этим свойством. Тогда равенство (2.15) можно переписать в виде

$$
\left.(\bar{A}-\alpha)\right|_{\mathscr{L}^{+}}=Q^{-1}[1+M(\alpha)](\bar{S}-\alpha) Q, \quad M(\alpha):=V\left[1+(\alpha-\mu)(\bar{S}-\alpha)^{-1}\right] .
$$

Значения оператор-функции $M(\alpha)$ - компактные операторы, а при больших положительных $\alpha$ оператор $1+M(\alpha)$ обратим. В силу аналитической теоремы Фредгольма [9, гл. 1] спектр оператора $1+M(\alpha)$ (а потому и спектр сужения $\left.A_{+}=\left.\bar{A}\right|_{L_{+}}\right)$в открытой правой полуплоскости может состоять только из изолированных собственных значений конечной алгебраической кратности.

Заметим, что $\operatorname{Im}\left[A x_{0}, x_{0}\right]=\left(\operatorname{Im} \alpha_{0}\right)\left[x_{0}, x_{0}\right]$, если $A x_{0}=\alpha_{0} x_{0}$. Так как $A$ диссипативен в пространстве $(\mathscr{H},[\cdot, \cdot])$, а $\mathscr{L}$ - неотрицательное подпространство, то число $\alpha \in \mathbb{C}$ может быть собственным значением оператора $A \mid \mathscr{L}$ только в случае $\operatorname{Im}\left[A x_{0}, x_{0}\right]=\left[x_{0}, x_{0}\right]=0$. Поэтому, если $A$ (или $\left.\left.A\right|_{\mathscr{L}}\right)$ строго диссипативен, то $\left.A\right|_{\mathscr{L}}$ не имеет собственных значений в $\mathbb{C}^{+}$, а потому вся эта полуплоскость принадлежит резольвентному множеству. Тем самым теорема полностью доказана для строго диссипативных операторов $A$.

В общем случае рассмотрим семейство операторов

$$
A_{\varepsilon}=A-\varepsilon P_{+}, \quad \varepsilon>0 .
$$

Уже доказано, что для этих операторов имеются максимальные неотрицательные $A_{\varepsilon}$-инвариантные подпространства с угловыми операторами $K_{\varepsilon}$. Сужения операторов $\bar{A}_{\varepsilon}$ на эти инвариантные подпространства являются строго диссипативными операторами, так как

$$
\operatorname{Re}\left[\left(A-\varepsilon P_{+}\right)\left(\begin{array}{c}
x_{+} \\
K x_{+}
\end{array}\right),\left(\begin{array}{c}
x_{+} \\
K x_{+}
\end{array}\right)\right] \leqslant-\varepsilon\left(x_{+}, x_{+}\right) .
$$

Поэтому открытая правая полуплоскость лежит в резольвентных множествах этих сужений. Эти сужения имеют представления

$$
\left.\bar{A}_{\varepsilon}\right|_{\mathscr{L}_{\varepsilon}^{+}}=Q^{-1}\left(\bar{S}+i \varepsilon+V_{\epsilon}(\bar{S}+i \varepsilon-\mu)\right) Q, \quad V_{\epsilon}:=G\left(1-K_{\varepsilon} G\right)^{-1} K_{\varepsilon} .
$$

Запишем модифицированное уравнение Риккати для оператора $A_{\varepsilon}$ :

$$
F+K_{\varepsilon}=\left(A_{22}-\mu\right)^{-1}\left(1-K_{\varepsilon} G\right)^{-1} K_{\varepsilon}(S+i \varepsilon-\mu) .
$$

Выберем подпоследовательность $\varepsilon_{n} \rightarrow 0$ так, что $K_{\varepsilon_{n}}=: K_{n} \rightarrow K_{0}$. Как и раньше, мы снова можем перейти к слабому пределу в этом уравнении. Тогда $K_{0}$ является угловым оператором $A$-инвариантного граф-подпространства $\mathscr{L}^{+}$. Сужение оператора $A$ на это инвариантное подпространство имеет представление (2.17), в котором нужно положить $\varepsilon=0$. Воспользуемся леммой 2.11, положив

$$
T=\bar{S}, \quad B=\bar{S}-\mu, \quad D_{n}=\left(1-K_{n} G\right)^{-1} K_{n}, \quad E_{n}=i \varepsilon_{n}\left(1+G D_{n}\right) .
$$

Условия этой леммы выполнены, поскольку $G$ компактен и $D_{n} \rightarrow D=(1-$ $\left.K_{0} G\right)^{-1} K_{0}$. Получаем, что правая полуплоскость свободна от спектра предельного оператора $T+G D B$, который подобен сужению оператора $A$ на $\mathscr{L}^{+}$. Теорема доказана. 
Замечание 2.13. Мы можем уточнить утверждение теоремы 2.1 для случая, когда оператор $A$ является диссипативным, но не $m$-диссипативным. Для этого удобно использовать язык теории полуфредгольмовых операторов (см. [14, гл. IV,$\S 5])$. Число $d=\operatorname{def}(J A-\alpha) \leqslant \infty$, равное размерности ядра $\operatorname{ker}\left(A^{*} J-\bar{\alpha}\right)$, не зависит от $\alpha \in \mathbb{C}^{+}$. В силу леммы 2.3 имеем

$\left.\operatorname{def}(J A-\alpha)\right|_{\alpha \in \mathbb{C}^{+}}=\left.\operatorname{def}(J A+\mu)\right|_{\mu \in \mathbb{C}^{-}}=\left.\operatorname{def}(\bar{S}(\mu)-\alpha)\right|_{\alpha=-\mu}=\left.\operatorname{def}(\bar{S}(\mu)-\alpha)\right|_{\alpha \in \mathbb{C}^{+}}$. Из второй теоремы устойчивости для полуфредгольмовых операторов при $\alpha \in$ $\mathbb{C}^{+}$получаем равенство

$$
\operatorname{ind}(\bar{S}-\alpha)=\operatorname{ind}(\bar{S}-\alpha+V(\bar{S}+\mu))=\operatorname{ind}\left(\left.A\right|_{\mathscr{L}}-\alpha\right)=d
$$

для индексов операторов. При этом число $\operatorname{nul}\left(\left.A\right|_{\mathscr{L}}-\alpha\right)$, равное размерности ядра $\operatorname{ker}\left(\left.A\right|_{\mathscr{L}}-\alpha\right)$, равно нулю для всех $\alpha \in \mathbb{C}^{+}$, за возможным исключением множества изолированных точек (см. подробнее [14, гл. IV, §5.6]).

\section{§3. Полугрупповые свойства сужений}

Мы предполагаем, что читателю известны определения голоморфной полугруппы и $C_{0}$-полугруппы и их генераторов (см., например, [8, I, II], [14, IX]) в гильбертовом (или банаховом) пространстве $\mathscr{H}$. Далее мы будем использовать только свойства резольвент генераторов таких полугрупп. Напомним следующие предложения. Первые два хорошо известны (см. цитированные монографии), они дают критерии того, что оператор является генератором $C_{0}$-полугруппы и голоморфной полугруппы соответственно, в терминах резольвенты генератора.

Теорема о генераторе $\boldsymbol{C}_{0}$-полугруппы (Феллер-Миядера-Филлипс). 3aмкнутый плотно определенный оператор $T: \mathscr{H} \rightarrow \mathscr{H}$ является генератором $C_{0}$-полугруппь тогда и только тогда, когда найдется число $\omega \in \mathbb{R}$, такое, что полуплоскость $\operatorname{Re} \lambda>\omega$ лежит в резольвентном множестве $\rho(T)$ и для всех цельх $n \geqslant 1$ u $\lambda>\omega$

$$
\left\|(T-\lambda)^{-n}\right\| \leqslant C(\lambda-\omega)^{-n},
$$

где постоянная $C$ не зависит от $\lambda u n$.

В этом случае для полугруппы $U(t)=\exp (T t)$ выполняется оценка $\|U(t)\| \leqslant$ $C e^{\omega t}$. Нижняя грань чисел $\omega$, для которых выполнена эта оценка или оценка (3.1), называется экспоненииальным типом $C_{0}$-полугруппы. В случае $\omega<0$ полугруппу называют экспоненииально устойчивой.

Теорема о генераторе голоморфной полугруппы. Замкнутый nлотно определенный оператор $T: \mathscr{H} \rightarrow \mathscr{H}$ является генератором голоморфной полугруппы тогда и только тогда, когда правая полуплоскость лежит в резольвентном множестве $\rho(T)$ и для всех $\lambda, \operatorname{Re} \lambda>0$, выполняется оченка

$$
\left\|(T-\lambda)^{-1}\right\| \leqslant C|\lambda|^{-1},
$$

где постоянная $C$ не зависит от $\lambda$.

Конечно, такая оценка выполняется, если $T$ является максимальным секториальным оператором, т.е. его числовой образ (множество значений квадратичной формы $(T x, x)$, когда $x$ пробегает $D(T)$ и $\|x\|=1)$ лежит в секторе $\Lambda_{\alpha}=\{\lambda:|\arg (\lambda-\pi)|<\alpha\}$ при некотором $\alpha<\pi / 2$. В этом случае неравенство (3.2) выполняется при $C=(\cos \alpha)^{-1}$. 
Далее удобно будет ввести следующее определение: полугруппу $U(t)=$ $\exp (T t)$ назовем квазиголоморфной, если для резольвенты ее генератора выполнена оценка (3.2) для всех $\lambda, \operatorname{Re} \lambda>\varepsilon$, где $\varepsilon>0$ произвольно, а постоянная $C$ зависит только $\varepsilon$. Очевидно, это условие эквивалентно тому, что $T-\varepsilon$ является генератором голоморфной полугруппы при любом $\varepsilon>0$. Известно, что квазиголоморфная полугруппа является $C_{0}$-полугруппой экспоненциального типа 0. Обратное, конечно, неверно.

Заметим, что в основной теореме 2.1 в явном виде не фигурировали условия на оператор $A_{11}$. Но получить результаты о полугрупповых свойствах сужения $A_{+}$без дополнительных условий на оператор $A_{11}$ - задача трудная. Мы вернемся к ней в другой работе, а здесь приведем результат для случая, когда оператор $A_{12}$ ограничен. Кроме того, мы рассмотрим случай, когда один из операторов $A_{11}$ или $S=S(\mu)$ генерирует квазиголоморфную полугруппу. Нам понадобятся два утверждения.

Теорема Герхарта [8, теорема V.1.11]. Экспоненциальный muп $C_{0}$-nолугруппь, генерируемой оператором $T$ в гильбертовом пространстве, совпадает с нижней гранъю чисел $\beta$, для которых полуплоскость $\operatorname{Re} \lambda>\beta$ лежит в $\rho(T) u$

$$
\sup _{\operatorname{Re} \lambda>\beta}\left\|(T-\lambda)^{-1}\right\|<\infty .
$$

Лемма 3.1. Пусть $T$ есть $m$-диссипативный, $a V$ - компактный операторы в $\mathscr{H}$. Тогда при любом $\varepsilon>0$ найдется достаточно большое число $r=r(\varepsilon)$, такое, что для всех $\lambda$ из полуплоскости $\operatorname{Re} \lambda \geqslant \varepsilon$, удовлетворяющих условию $|\lambda|>r$, оператор $T+V-\lambda$ обратим $u$

$$
\left\|(T+V-\lambda)^{-1}\right\| \leqslant(2 \varepsilon)^{-1} .
$$

Доказательство. Действуем так же, как в лемме 2.7. Пусть сначала $V=$ $(\cdot, \varphi) \psi$ - одномерный оператор в $\mathscr{H}$. Представим его в виде

$$
V=V_{0}+V_{1}, \quad V_{0}=(\cdot, \varphi) \psi_{0}, \quad\left\|V_{1}\right\|<\delta, \quad \psi_{0} \in \mathscr{D}(T) .
$$

Положим $\psi_{1}=(T-1) \psi_{0}$. Тогда

$$
(T-\lambda)^{-1} \psi_{0}=(T-\lambda)^{-1}(T-1)^{-1} \psi_{1}=(\lambda-1)^{-1}\left[(T-\lambda)^{-1} \psi_{1}-(T-1)^{-1} \psi_{1}\right] .
$$

Фиксируем $\varepsilon>0$ и возьмем $\delta<\varepsilon / 4$. В полуплоскости $\operatorname{Re} \lambda \geqslant \varepsilon$ имеем оценку $\left\|(T-\lambda)^{-1}\right\| \leqslant \varepsilon^{-1}$. Поэтому при таких $\lambda$

$\left\|(T-\lambda)^{-1}\left(V_{1}+V_{0}\right)\right\| \leqslant \frac{\delta}{\varepsilon}+\frac{\|\varphi\|\left\|\psi_{1}\right\|}{|\lambda-1|}\left(\frac{1}{\varepsilon}+1\right)<\frac{1}{2}, \quad$ если $|\lambda|>1+4\|\varphi\|\left\|\psi_{1}\right\| \frac{1+\varepsilon}{\varepsilon}$.

Очевидно, что из этой оценки следует (3.3). Доказательство сохраняется, если $V$ конечномерен. Так как компактный оператор можно приблизить по норме с произвольной точностью конечномерным, то оценка остается справедливой и для компактного оператора $V$.

Теорема 3.2. Пусть выполнены условия теоремы 2.1 и $A_{+}$-сужение $m$-диссипативного оператора $\bar{A}$ в пространстве $(\mathscr{H},[\cdot, \cdot])$ на инвариантное максимальное неотрицательное подпространство $\mathscr{L}^{+}$, такое, что $\sigma\left(A_{+}\right)$лежит в замкнутой левой полуплоскости. Пусть также выполнено одно из условий 
(i) оператор $A_{12}$ компактен;

(ii) оператор $A_{12}$ ограничен $и$

$$
\sup _{\mu \in \mathbb{C}^{-},|\mu|>r}\left\|\left(A_{22}-\mu\right)^{-1}\right\| \rightarrow 0 \quad \text { npu } r \rightarrow \infty .
$$

Тогда $A_{+}$генерирует $C_{0}$-полугруппу экспоненииального типа 0. Если, кроме того, $\bar{A}$ равномерно $m$-диссипативен в пространстве $(\mathscr{H},[\cdot, \cdot]), m . е . \operatorname{Re}[A x, x]$ $\leqslant-\varepsilon(x, x)$ для всех $x \in D(A)$ при некотором $\varepsilon>0$, то $A_{+}$генерирует экспоненииально устойчивую $C_{0}$-полугруппу.

Доказательство. Из формул (2.14) и (2.15) следует, что

$$
A_{+}:=\left.\bar{A}\right|_{\mathscr{L}^{+}}=Q^{-1} X Q, \quad X=\bar{S}+A_{12}(F+K) .
$$

Оператор $\bar{S}=\bar{S}(\mu)$ является $m$-диссипативным, а потому генерирует полугруппу сжатий в $\mathscr{H}^{+}$, тем более, $C_{0}$-полугруппу. Из теоремы устойчивости для $C_{0}$-полугрупп при ограниченных возмущениях [14, теорема IX.2.1] получаем, что оператор $X$ также генерирует $C_{0}$-полугруппу. Кроме того, спектр оператора $X$ лежит в замкнутой левой полуплоскости. Тогда, если оператор $A_{12}$ компактен, в силу леммы 3.1 резольвента $(X-\alpha)^{-1}$ является ограниченной в полуплоскости $\operatorname{Re} \alpha>\varepsilon$ при любом $\varepsilon>0$. Остается воспользоваться теоремой Герхарта.

Теперь пусть выполнено условие (ii) (отметим, что оно заведомо выполнено, если $-A_{22}$ генерирует квазиголоморфную полугруппу). Как и ранее, достаточно доказать ограниченность резольвенты $(X-\alpha)^{-1}$ в полуплоскости $\operatorname{Re} \alpha>\varepsilon$ при любом $\varepsilon>0$ и больших $|\alpha|>r(\varepsilon)$. Тогда вновь можно воспользоваться теоремой Герхарта.

Воспользуемся представлением (2.15), из которого получим

$$
(X-\alpha)^{-1}=(S-\alpha)^{-1}(1+M(\mu, \alpha))^{-1}, \quad M(\mu, \alpha):=V\left[1+(\alpha-\mu)(\bar{S}-\alpha)^{-1}\right] .
$$

Здесь $V=G(\mu)(1-K G(\mu))^{-1} K$. Так как оператор $A_{12}$ ограничен и выполнено условие $(3.4)$, то $\|V(\mu)\| \rightarrow 0$ при $\mu \rightarrow \infty$ в полуплоскости $\mathbb{C}^{-}$. Выбор числа $\mu$ в представлении (3.5) находится в нашей власти, положим $\mu=-\bar{\alpha}$. Из оценки $(\alpha+\bar{\alpha})\left\|(\bar{S}-\alpha)^{-1}\right\| \leqslant 2$ следует $\|M(-\bar{\alpha}, \alpha)\| \rightarrow 0$ при $\alpha \in \mathbb{C}^{+},|\alpha| \rightarrow \infty$. Из представления (3.5) получаем равномерную ограниченность резольвенты $(X-\alpha)^{-1}$ в полуплоскости $\operatorname{Re} \alpha \geqslant \varepsilon>0$ при больших $|\alpha|$. Поэтому экспоненциальный тип генерируемой оператором $X$ полугруппы неположителен.

Пусть теперь оператор $\bar{A}$ является равномерно $m$-диссипативным в пространстве $(\mathscr{H},[\cdot, \cdot])$. Тогда $J \bar{A}$ обратим и $\operatorname{Re}(J \bar{A} x, x) \geqslant \varepsilon(x, x)$ при некотором $\varepsilon>0$. Следовательно, оператор $J \bar{A}-\lambda J$ обратим в полосе $|\operatorname{Re} \lambda|<\varepsilon$, а в более узкой полосе $|\operatorname{Re} \lambda|<\delta<\varepsilon$ верна оценка

$$
\left\|\left(A_{+}-\lambda\right)^{-1}\right\| \leqslant\left\|(A-\lambda)^{-1}\right\|=\left\|(J A-\lambda J)^{-1}\right\| \leqslant 1 /(\varepsilon-\delta) .
$$

Из теоремы Герхарта получаем, что $A_{+}$генерирует полугруппу экспоненциального типа $\leqslant-\varepsilon$. Теорема доказана.

Теорема 3.3. Пусть выполнены условия теоремы 2.1 и $A_{+}$-сужение m-диссипативного оператора $\bar{A}$ в пространстве $(\mathscr{H},[\cdot, \cdot])$ на инвариантное, максимальное неотрицательное подпространство $\mathscr{L}^{+}$, такое, что $\sigma\left(A_{+}\right)$лежит в замкнутой левой полуплоскости. Пусть также выполнено одно из условий 
(i) оператор $\bar{S}=\bar{S}(\mu)$ генерирует квазиголоморфную полугруппу при некотором $\mu \in \mathbb{C}^{-}$;

(ii) оператор $\bar{A}_{11}$ генерирует квазиголоморфную полугруппу и при некотором $\mu \in \mathbb{C}^{-}$оператор $R(\mu):=A_{12}\left(A_{22}-\mu\right)^{-1} A_{21}$ является $A_{11}$-ограниченным с нулевой $A_{11}$-гранъю, т.е. при любом $\delta>0$ найдется $a=a(\delta)$, такое, что

$$
\|R(\mu) x\| \leqslant \delta\left\|A_{11} x\right\|+a\|x\|, \quad x \in \mathscr{D}\left(A_{11}\right) ;
$$

(iii) оператор $\bar{A}_{11}$ генерирует квазиголоморфную полугруппу, а оператор $A_{21}$ является $A_{11}$-ограниченным.

Тогда $A_{+}$генерирует квазиголоморфную полугруппу.

Доказательство. Пусть $\bar{S}\left(\mu_{0}\right)$ генерирует квазиголоморфную полугруппу. Из формулы

$$
R(\mu)=A_{12}\left(A_{22}-\mu\right)^{-1} A_{21}=R\left(\mu_{0}\right)+\left(\mu-\mu_{0}\right) G(\mu) F\left(\mu_{0}\right)
$$

следует, что при любом $\mu \in \mathbb{C}^{-}$оператор $S(\mu)$ является ограниченным возмущением оператора $S\left(\mu_{0}\right)$. Кроме того, $\bar{S}(\mu)$ является $m$-диссипативным, а потому он также генерирует квазиголоморфную полугруппу.

Воспользуемся равенством

$$
\bar{S}(\mu)-\alpha=\left[1-\left(\mu-\mu_{0}\right) G(\mu) F\left(\mu_{0}\right)\left(\bar{S}_{0}-\alpha\right)^{-1}\right]\left(\bar{S}_{0}-\alpha\right),
$$

где $\bar{S}_{0}=\bar{S}\left(\mu_{0}\right)$. Найдется достаточно большое число $r=r(\mu, \varepsilon)$, такое, что при $|\alpha|>r, \operatorname{Re} \alpha \geqslant \varepsilon$ выполняется оценка

$$
\left\|\left(\mu-\mu_{0}\right) G(\mu) F\left(\mu_{0}\right)\left(\bar{S}_{0}-\alpha\right)^{-1}\right\|<1 / 2 .
$$

Следовательно,

$$
\left\|(\bar{S}(\mu)-\alpha)^{-1}\right\|<2\left\|\left(\bar{S}_{0}-\alpha\right)^{-1}\right\|<C(\varepsilon)|\alpha|^{-1} \quad \text { при }|\alpha|>r(\mu, \varepsilon), \operatorname{Re} \alpha \geqslant \varepsilon .
$$

В этом неравенстве постоянная $C=C(\varepsilon) \geqslant 1$ от $\mu$ не зависит. Фиксируем $\varepsilon>0$ и выберем $\mu<0$ так, чтобы

$$
\left\|G(\mu)(1-K G(\mu))^{-1}\right\|<(4 C)^{-1} .
$$

Тогда для функции $M(\mu, \alpha)$, определенной в $(3.5)$, получаем оценку

$$
\|M(\mu, \alpha)\|<(4 C)^{-1}\left(1+C\left(1+|\mu||\alpha|^{-1}\right)<3 / 4,\right.
$$

если $|\alpha|>\max \{r,|\mu|\}$. Так как $\bar{S}(\mu)$ генерирует квазиголоморфную полугруппу, то из представления (3.5) имеем

$$
\left\|(X-\alpha)^{-1}\right\| \leqslant 4\left\|(\bar{S}(\mu)-\alpha)^{-1}\right\| \leqslant C|\alpha|^{-1} \quad \text { при } \operatorname{Re} \alpha \geqslant \varepsilon,|\alpha|>r(\mu, \varepsilon),
$$

с постоянной $C$, не зависящей от $\alpha$. Так как спектр оператора $X$ лежит в левой полуплоскости, то эта оценка остается справедливой во всей полуплоскости $\operatorname{Re} \alpha \geqslant \varepsilon$. Следовательно, $X$ генерирует квазиголоморфную полугруппу.

Покажем, что из условия (ii) вытекает условие (i). По условию $\left.\| \bar{A}_{11}-\alpha\right)^{-1} \| \leqslant$ $C(\varepsilon)|\alpha|^{-1}$ при $\operatorname{Re} \alpha \geqslant \varepsilon$, поэтому из второго условия в (ii) при тех же значениях $\alpha$ получаем

$$
\begin{aligned}
\left\|R(\mu)\left(\bar{A}_{11}-\alpha\right)^{-1} x\right\| & \leqslant \delta\left\|\bar{A}_{11}\left(\bar{A}_{11}-\alpha\right)^{-1} x\right\|+a\left\|\left(\bar{A}_{11}-\alpha\right)^{-1} x\right\| \\
& \leqslant\left(\delta(1+C(\varepsilon))+a C(\varepsilon)|\alpha|^{-1}\right)\|x\| .
\end{aligned}
$$


Следовательно, при $\delta<(2+2 C(\varepsilon))^{-1}$ и достаточно больших $|\alpha|$ в полуплоскости $\operatorname{Re} \alpha \geqslant \varepsilon$ выполнена оценка $\left\|R(\mu)\left(\bar{A}_{11}-\alpha\right)^{-1}\right\|<1 / 2$. Из равенства

$$
(\bar{S}-\alpha)^{-1}=\left[1+R(\mu)\left(\bar{A}_{11}-\alpha\right)^{-1}\right]^{-1}\left(\bar{A}_{11}-\alpha\right)^{-1}
$$

и $m$-диссипативности оператора $\bar{S}=\bar{S}(\mu)$ получаем, что $\bar{S}$ генерирует квазиголоморфную полугруппу.

Покажем, что из условия (iii) следует (ii). Имеем $R(\mu)=G(\mu) A_{21}$. Но $G(\mu)$ компактен; поэтому если $A_{21}$ является $A_{11}$-ограниченным, то $R(\mu)$ обладает тем же свойством с нулевой $A_{11}$-гранью. Теорема полностью доказана.

Автор признателен проф. Т. Я. Азизову и проф. А. М. Гомилко за кропотливый труд при чтении рукописи работы. Их замечания позволили устранить ряд погрешностей в рукописи и улучшить изложение.

\section{ЛитерАтУРА}

[1] F. V. Atkinson, H. Langer, R. Mennicken, A. A. Shkalikov, The essential spectrum of some matrix operators, Math. Nachr., 167 (1994), 5-20.

[2] Т. Я. Азизов, Инвариантные подпространства и критерии полноты системы собственных векторов J-диссипативных операторов в пространстве Понтрягина, Докл. АН СССР, 200:5 (1971), 1015-1017.

[3] Т. Я. Азизов, Диссипативные операторь в гилъбертовом пространстве с индефинитной метрикой, Изв. АН СССР. Сер. матем., 37:3 (1973), 639-662.

[4] Т. Я. Азизов, С. А. Хорошавин, Об инвариантных подпространствах операторов, действующих в пространстве с индефинитной метрикой, Функц. анализ и его прил., 14:4 (1980), 1-7.

[5] Т. Я. Азизов, И. С. Иохвидов, Основы теории линейных операторов в пространствах с индефинитной метрикой, Наука, М., 1986.

[6] M. A. Dritschel, A method for constructing invariant subspaces for some operators on Krein spaces, in: Operator Theory: Advances and Applications, vol. 61, Birkhäuser, Basel-Boston, 1993, 85-113.

[7] M. A. Dritschel, Compact perturbations of operators on Krein spaces, in: Contemporary Mathematics, vol. 189, 1995, 201-211.

[8] K. J. Engel, R. Nagel, One-parameter Semigroups for Linear Evolution Equations, Springer-Verlag, Berlin-Heidelberg-New York, 2000.

[9] И. Ц. Гохберг, М. Г. Крейн, Введение в теорию несамосопряженных операторов в гильбертовом пространстве, Наука, М., 1965.

[10] А. М. Гомилко, Инвариантные подпространства J-диссипативных операторов, Функц. анализ и его прил., 19:3 (1985), 61-62.

[11] П. Халмош, Гильбертово пространство в задачах, Мир, М., 1970.

[12] P. Jonas, Compact perturbations of definitizable operators. II, J. Operator Theory, 8:1 (1982), 3-18.

[13] P. Jonas, On a class of self-adjoint operators in Krein space and their compact perturbations, Integral Equations Operator Theory, 11:3 (1988), 351-384.

[14] Т. Като, Теория возмущений линейных операторов, Мир, М., 1972.

[15] А. Г. Костюченко, М. Б. Оразов, О некоторых свойствах корней самосопряженного квадратичного пучка, Функц. анализ и его прил., 9:4 (1975), 28-40.

[16] М. Г. Крейн, Об одном приложении принципа неподвижной точки в теории операторов в пространстве с индефинитной метрикой, УМН, 50 (1950), 180190. 
[17] М. Г. Крейн, Об одном новом применении приниипа неподвижной точки в теории операторов в пространстве с индефинитной метрикой, Докл. АН СССР, 154:5 (1964), 1023-1026.

[18] М. Г. Крейн, Г. К. Лангер, О дефинитных пространствах и обобщенных резольвентах эрмитова оператора в пространстве $\Pi_{\varkappa}$, Функц. анализ и его прил., $\mathbf{5 : 2}$ (1971), 59-71; 5:3 (1971), 54-69.

[19] Г. К. Лангер, O J-эрмитовых операторах, Докл. АН СССР, 134:2 (1962), 263-266.

[20] H. Langer, Eine Veralgemeinerung eines Satzes von L. S. Pontrjagin, Math. Ann., 152:5 (1963), 434-436.

[21] H. Langer, Invariant subspaces for a class of operators in spaces with indefinite metric, J. Funct. Anal., 19:3 (1975), 232-241.

[22] H. Langer, Spectral functions of definitizable operators in Krein spaces, Lecture Notes in Math., 948 (1982), 1-46.

[23] R. Mennicken, A. A. Shkalikov, Spectral decomposition of symmetric operator matrices, Math. Nachr., 179 (1996), 259-273.

[24] Л. С. Понтрягин, Эрмитовы операторы в пространствах с индефинитной метрикой, Изв. АН СССР. Сер. матем., 8 (1944), 243-280.

[25] А. А. Шкаликов, О существовании инвариантных подпространств у диссипативных операторов в пространстве Крейна, Фундам. прикл. матем., 5:2 (1999), $627-635$.

[26] А. А. Шкаликов, О принципах отбора и свойствах некоторых частей собственных и присоединенных элементов операторных пучков, Вестник МГУ. Матем. Mex., 43:4 (1988), 16-25.

[27] А. А. Шкаликов, Об инвариантных подпространствах диссипативных операторов в пространстве с индефинитной метрикой, Труды МИРАН, 248 (2005), 294-303.

[28] С. Л. Соболев, Движение симметрического волчка с полостъю, заполненной жидкостъю, Жуурнал прикл. матем. и техн. физики, 1960, № 3, 20-55.

Московский государственный университет им. М. В. Ломоносова, Поступило в редакцию механико-математический факультет email: ashkalikov@yahoo.com 